\title{
Effects of Vitamin K3 Combined with UVB on the Proliferation and Apoptosis of Cutaneous Squamous Cell Carcinoma A43I Cells
}

This article was published in the following Dove Press journal: OncoTargets and Therapy

\author{
Shangyuchen Shi ${ }^{1, *}$ \\ Gang Zheng ${ }^{2,3, *}$ \\ Chunsheng Yang ${ }^{4, *}$ \\ Xi Chen ${ }^{2}$ \\ Qiuyue Yan' \\ Fan Jiang' \\ Xiaojie Jiang' \\ Yong Xin' \\ Guan Jiang ${ }^{2}$
}

'Department of Radiotherapy, Affiliated Hospital of Xuzhou Medical University, Xuzhou 221002, People's Republic of China; ${ }^{2}$ Department of Dermatology, Affiliated Hospital of Xuzhou Medical University, Xuzhou 221002, People's Republic of China; ${ }^{3}$ Department of Dermatology, Xuzhou TCM Hospital Affiliated to Nanjing University of Chinese Medicine, Huai'an 221003 , People's Republic of China; ${ }^{4}$ The Affiliated Huai'an Hospital of Xuzhou Medical University, The Second People's Hospital of Huai'an, Huai'an 223002,

People's Republic of China

*These authors contributed equally to this work
Purpose: Cutaneous squamous cell carcinoma (cSCC) is the second most common form of skin cancer and its incidence continues to rise yearly. Photodynamic therapy (PDT) is a noninvasive form of cancer therapy, which utilizes the combined action of a photosensitizer, light, and oxygen molecules to selectively cause cellular damage to tumor cells. Vitamin K3 (VitK3) has been shown to induce apoptosis and inhibit the growth of tumor cells in humans. The purpose of this study was to determine the effect of VitK3 and ultraviolet radiation B (UVB) on oxidative damage, proliferation and apoptosis of A431 cells.

Methods: CCK-8 assay was used to detect cell proliferation; Hoechst staining, TUNEL assay and flow cytometry analysis were used to detect apoptosis. Western Blot was perfomed to measure the expression of apoptosis-related proteins. Flow cytometry analysis was employed to detect the reactive oxygen species (ROS) levels and mitochondrial membrane potential. Finally, the role of VitK3 in combination with UVB on the proliferation and apoptosis of A431 cells was investigated using mice xenograft models.

Results: We found that the co-treatment of VitK3 combined with UVB more significantly inhibited the growth and proliferation of A431 cells than either VitK3 or UVB alone. Hoechst 33258 staining and flow cytometry analysis revealed that apoptosis was more pronounced in the VitK3-UVB group compared to the VitK3 and UVB groups. Moreover, flow cytometry analysis showed that ROS and the depolarization of the mitochondrial membrane potential were higher in all the co-treatment groups compared to the control, VitK3, and UVB groups. The VitK3-UVB group exhibited a significantly lower tumor growth rate in mouse xenograft models.

Conclusion: This study reveals that VitK3 combined with UVB inhibits the growth and induces apoptosis of A431 cells in vitro and suppresses tumor growth and promotes apoptosis of cSCC in vivo.

Keywords: photodynamic, photosensitizer, oxidative, depolarization

\section{Introduction}

Cutaneous squamous cell carcinoma (cSCC), also known as Squamous cell carcinoma, is a malignant tumor arising from the keratinocytes in the skin epidermis or appendages. ${ }^{1}$ cSCC accounts for $20 \%$ of non-melanoma skin cancers while basal cell carcinoma accounts for $80 \% .^{2}$ Recent epidemiological data shows that there is an upward trend in the $\mathrm{cSCC}$ to $\mathrm{BCC}$ ratio. ${ }^{3}$ Distant metastasis occurs at the time of the diagnosis in approximately $5 \%$ of the patients with high-risk cSCC due to early occurrence of hematological and lymphoid metastasis. ${ }^{4}$ For already diagnosed

Guan Jiang

Email dr.guanjiang@gmail.com 
patients, the main treatments modalities for cSCC include surgery, radiotherapy, local and systemic chemotherapy, laser therapy, photodynamic therapy (PDT), gene therapy, and liquid nitrogen cryotherapy. ${ }^{5}$

PDT is a form of non-invasive tumor therapy that combines the action of a photosensitizer, light of appropriate wavelength and oxygen molecules to selectively kill malignant lesions. Moreover, a large number of reactive oxygen species (ROS) produced in PDT destroy tumor cells and thus inhibits tumor growth. ${ }^{6}$ Since the 1980s, PDT has attracted the attention of scholars due to its wide application in various tumor types, it does not exhibit drug resistance, it is less toxic to normal cells and causes minimal injury to the surrounding normal tissue. There are several possible mechanisms through which PDT eliminates tumor cells: 1) during PDT treatment, a large number of singlet oxygen and reactive oxygen species (ROS) are produced after the depolarization of the mitochondrial membrane and this leads to damage of essential macromolecules and finally results in cell apoptosis or tissue necrosis; 2) cSCC is a tumor with its own blood supply. Following PDT, the endothelial cells of the tumor blood vessels are damaged, and their mechanical repair may lead to vascular stenosis, embolism, and further reduction or inhibition of blood supply to the tumor cells; 3 ) in the process of killing tumor cells, PDT increases the expression and presentation of tumor antigens, which enhance the immune response and induce immunity to tumor cells; 4) there are also other several mechanisms with combined effects that cause tumor killing. ${ }^{7,8}$ Recently, PDT has received significant results in the treatment of skin cancer and precancerous lesions (e.g., basal cell carcinoma [BCC], cSCC, solar keratosis, and Bowen's disease). ${ }^{9}$

Traditionally, photosensitizers were mainly divided into first- and second-generation photosensitizers. As a first-generation photosensitizer, photofrin has been used in the treatment of lung cancer, bladder cancer, esophageal cancer, and early cervical cancer. However, photofrin has been associated with low specificity, prolonged stay in blood circulation, short absorption wavelength, and high levels of skin toxicity. Moreover, its hydrophobicity makes it difficult to metabolize, and this inevitably damages other tissues. ${ }^{10}$ The second-generation photosensitizers primarily include porphyrin derivatives, chlorophyll, and other compounds, which can be cleared more efficiently in normal tissues and plasma and are relatively enriched in the tumor tissues to improve the therapeutic effect. ${ }^{11}$ However, the selectivity of second-generation photosensitizers is weak, and the concentration in the tumor is not optimal hence they do not meet the clinical requirements. Therefore, recent efforts have focused on improving the quality of photosensitizers to enhance their treatment efficacy. Vitamin K3 (VitK3), also known as menadione (2-Methyl-1, 4-naphthoquinon), has a chemical structure as illustrated in Figure 1A. It is a synthetic coagulant, which is readily soluble in water and hot ethanol, and decomposes easily. Clinically, it is often used as a hemostatic drug. Recently, VitK3 has been reported to induce apoptosis and inhibit the growth of tumor cells, ${ }^{12,13}$ which suggests its involvement in oxidative stress. Bergeron et $a l,{ }^{14}$ reported that VitK3 could be used as a radiosensitizer for hypoxic tumors, whereas $\mathrm{Xu}$ et $\mathrm{al}^{15}$ reported that VitK3 could be used as a photosensitizer. Therefore, the aim of this study was to determine the effect of VitK3 as a potential photosensitizer combined with UVB on apoptosis of cutaneous squamous cell carcinoma A431 cells.

\section{Materials and Methods Cell Lines and Culture Conditions}

The A431 cell line was purchased from the cell bank of the Chinese Academy of Sciences. DMEM medium and $10 \%$ fetal bovine serum (FBS) without mycoplasma was bought from GIBCO (US). A431 cells were cultured in complete DMEM medium (containing 10\% FBS without mycoplasma) and cultured in a cell incubator containing $5 \% \mathrm{CO}_{2}$ at $37^{\circ} \mathrm{C}$.

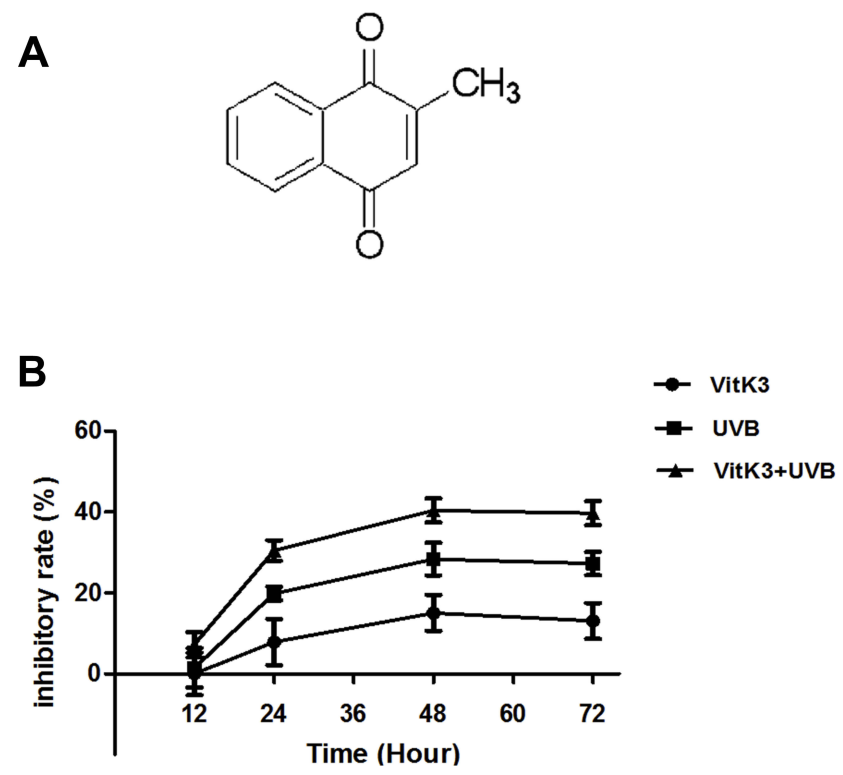

Figure I The chemical structure of vitamin $\mathrm{K} 3(\mathbf{A})$. Comparison of inhibition rate on cellular proliferation of A43I cells between VitK3-UVB group and individual treatment groups $(\mathbf{B})$. 


\section{Cell Proliferation Assay}

To assess the effects of VitK3 (Sigma) on the proliferation rate of A431 cells, the cells were seeded into 96-well plates (Constar, USA) at a density of $1 \times 10^{4}$ cells/well and treated with various concentrations of VitK 3 (30, 45, 60 , and $100 \mu \mathrm{mol} / \mathrm{L})$. The same method was used to determine the effect of UVB irradiation on cell proliferation. The A431 cells were irradiated with $0.5,1.0,1.5$, and $2.0 \mathrm{~J} / \mathrm{cm}^{2}$ in the presence of different doses of UVB. After culture for $24 \mathrm{hrs}, 10 \mu \mathrm{L}$ of the CCK-8 solution (Vicmed, Xuzhou, China) was added to each well, followed by incubation for $2 \mathrm{hrs}$ at $37^{\circ} \mathrm{C}$. The inhibition rate of cell growth was calculated and used to plot the inhibition ratedrug concentration action time curve. Afterwards, the cells were divided into negative control group (no treatment), VitK3 group, UVB group, and VitK3-UVB group. Median inhibitory concentration (IC50) of VitK3 and the half inhibitory doses of UVB were selected for the drug concentration and light dose of the next experiment. Subsequent to VitK3 drug treatment, cells in all groups were subjected to a light avoidance preservation for $4 \mathrm{hrs}$, after which the UVB group and VitK3-UVB group were exposed to UVB irradiation. The optical density (OD value) of the cells media was measured at $450 \mathrm{~nm}$ using M $\mu l$ tiskan Spectruman (Thermo Scientific, Finland) at $12 \mathrm{hrs}, 24 \mathrm{hrs}, 48 \mathrm{hrs}$, and $72 \mathrm{hrs}$.

\section{Hoechst 33258 Staining}

Cells were seeded in six-well plates at a density of $2 \times 10^{5}$ cells/well. According to the CCK8 assay results, median inhibitory concentration (IC50) of VitK3 $(40 \mu \mathrm{mol} / \mathrm{L})$ and the half inhibitory doses of UVB $\left(800 \mathrm{~mJ} / \mathrm{cm}^{2}\right)$ were selected for the drug treatment concentration and light dose. Then, cells were divided into negative control group (no treatment), VitK3 group $(40 \mu \mathrm{mol} / \mathrm{L})$, UVB group $\left(800 \mathrm{~mJ} / \mathrm{cm}^{2}\right)$, and VitK3-UVB group (VitK3 40 $\mu \mathrm{mol} / \mathrm{L}$ and UVB $800 \mathrm{~mJ} / \mathrm{cm}^{2}$ ). Next, VitK3 was added to cells of VitK3 group and VitK3-UVB group, and all the groups were subjected to light avoidance preservation for $4 \mathrm{hrs}$. Afterwards, cells in the UVB group and VitK3-UVB group were exposed to UVB irradiation. They were naturally dried and subsequently immersed in a $4 \%$ paraformaldehyde fixation solution for $30 \mathrm{~min}$, dripped in a staining solution, and incubated at room temperature for $10 \mathrm{~min}$. The cells were washed in PBS buffer and activated with ultraviolet light at $340 \mathrm{~nm}$. Finally, photos were taken using a fluorescent microscope.

\section{Flow Cytometry Analysis}

Apoptosis was assessed using Annexin-V FITC/PI staining. A431 cells in good condition were seeded in six-well plates at a density of $5 \times 10^{5}$ cells/well. They were grouped and treated similar to the CCK8 assay and Hoechst 33258 Staining assay. After digestion and centrifugation, the cells rinsed with PBS (KeyGEN BioTECH Corp., Ltd, Jiangsu, China) 2 times. Next, the cells were suspended in $500 \mu \mathrm{L}$ Binding Buffer, and treated with $5 \mu \mathrm{L}$ Annexin V-FITC (KeyGEN BioTECH Corp., Ltd, Jiangsu, China) and $5 \mu \mathrm{L}$ Propidium Iodide (KeyGEN BioTECH Corp., Ltd, Jiangsu, China) at room temperature. The cells were incubated for 5-15 min in darkness. Finally, apoptosis rate was detected by flow cytometry.

To measure the production of reactive oxygen species (ROS) in the cells, cells were seeded in six-well plates at a density of $2 \times 10^{5}$ cells/well. They were then divided into different treatment groups as described above sections. The DCFH-DA was diluted in a serum-free medium to a final concentration of $10 \mu \mathrm{M}$. The cells were suspended in diluted DCFH-DA and then incubated at $37^{\circ} \mathrm{C}$ for $20 \mathrm{~min}$. The cells were washed with serum-free cell culture medium to remove free DCFH-DA. Finally, the level of ROS in cells was detected by flow cytometry (Becton-Dickinson FACS Calibur, USA) $(\mathrm{Ex}=488 \mathrm{~nm} ; \mathrm{Em}=530 \mathrm{~nm})$.

To measure the mitochondrial membrane potential (MMP), cells were seeded in six-well plates at a density of $1 \times 10^{6}$ cells/well, and then grouped as described above. They were subsequently put in $500 \mu \mathrm{L} \mathrm{JC}-1$ solution to form a homegous suspension and incubated at $37^{\circ} \mathrm{C}$ for 15 to $20 \mathrm{~min}$. The cells were collected by centrifugation, and resuspended in incubation buffer for $60 \mathrm{~min}$ at $37^{\circ} \mathrm{C}$ in a $5 \% \mathrm{CO}_{2}$ incubator. Finally, flow cytometry was performed to measure MMP level.

\section{Western Blot Analysis}

Cells were lysed with $1 \mathrm{~mL}$ ice-cold lysis buffer $(1 \%$ triton x-100, $20 \mathrm{~mm}$ Tris-Hcl, ph 7.5, $150 \mathrm{~mm} \mathrm{NaCl}, 10 \mathrm{~mm}$ naf, $1 \mathrm{~mm}$ Na3vo4, $10 \mathrm{~mm}$ PMSF, $1 \mathrm{~mm}$ benzaminidine, $5 \mathrm{mg} / \mathrm{mL}$ aprotinin, $3 \mathrm{mg} / \mathrm{mL}$ pepstatin, $5 \mathrm{mg} / \mathrm{mL}$ leupeptin). The cell lysate was centrifuged at $15,000 \mathrm{rpm}, 4^{\circ} \mathrm{C}$ for $20 \mathrm{~min}$. Next, the protein concentration was measured using BCA protein assay (Beyotime, Beijing, China). The proteins were heated at $100{ }^{\circ} \mathrm{C}$ for $10 \mathrm{~min}$, and then equal amount of protein per group was resolved on $10 \%$ SDSPAGE gel. Next, the proteins were transferred to nitrocellulose (NC) membranes. Thereafter, the membranes were 
blocked with 5\% skim milk for $2 \mathrm{hrs}$ at room temperature and incubated with the following primary antibodies: rabbit anti-Caspase-3 (1:500 dilution; Proteintech, America), antiCaspase-9 (1:300 dilution; Proteintech, Wuhan, China), anti-Bcl-2 (1:1000 dilution; Proteintech, America), antiBax (1:6000 dilution; Proteintech, Wuhan, China), anti-Cytc (1:5000 dilution; ABCAM, Britain), and mouse anti- $\beta$-actin (1:2000 dilution; Cell Signaling Technology, Shanghai, China). Subsequently, the NC membranes were washed and incubated with the corresponding goat antimouse or anti-rabbit IgG-HRP (Bioworld Technology, China) at room temperature for $2 \mathrm{~h}$. Finally, the immunoreactive signals were detected with SuperSignal ECL (Pierce, Rockford, IL, USA).

\section{Tumor Xenograft Study}

All in vivo experiments were approved by the Animal Ethics Committee of Xuzhou Medical University, and complied with the guidelines of the Laboratory Animal Ethical Committee of Xuzhou Medical University. Female BALB/c nude mice (four to five weeks old) were bought from Shanghai SLAC Laboratory Animal Co., Ltd. Briefly, $0.1 \mathrm{~mL}$ of human CSCC cell line, A431, was subcutaneously injected into the right armpit of nude mice at a dose of $1 \times 10^{7}$ cells $/ \mathrm{mL}$. When the tumor grew to $50-100 \mathrm{~mm}^{3}$, the mice were randomly divided into control group, VitK3 group, UVB group, and VitK3-UVB group with five mice per group. In the control group, $5 \mathrm{~mL} / \mathrm{kg}$ of the PBS solvent was injected into the tumor, and the drug was administered once; in the VitK3 group, VitK3 was injected once at a dose of $10 \mathrm{mg} / \mathrm{kg}$ into and around the tumors. In the UVB group, $5 \mathrm{~mL} / \mathrm{kg}$ of PBS solvent was injected into and around the tumor, and then UVB was administered for 20 min three times at one day intervals. In the VitK3-UVB group, $10 \mathrm{mg} / \mathrm{kg}$ of VitK3 was injected into the tumor, and UVB was administered for $20 \mathrm{~min}$ three times at one day intervals after $6 \mathrm{hrs}$. The tumor volume (V) was measured using a Vernier caliper. The relative tumor growth rate $(\mathrm{T} / \mathrm{C})$ and tumor weight inhibitory rate were calculated using the formula: $\mathrm{T} / \mathrm{C}(\%)=\mathrm{T}_{\mathrm{RTV}} / \mathrm{C}_{\mathrm{RTV}} \times 100$, tumor weight inhibitory rate $=\left(\mathrm{Wc}_{\mathrm{t}} \mathrm{W}_{\mathrm{t}}\right) / \mathrm{Wc} \times 100 \%(\mathrm{RTV}$ : the ratio of the mouse tumor weight at the time of sacrifice to the initial tumor weight, $\mathrm{T}_{\mathrm{RTV}}$ : mean of RTV for each treatment group, $\mathrm{C}_{\mathrm{RTV}}$ : mean of $\mathrm{RTV}$ for control group, Wc: average tumor weight in the control group, $\mathrm{W}_{\mathrm{t}}$ : average tumor weight in each treatment group). Mice were exposed to light three times after which two mice from each group were killed. After 20 days, all mice were killed and the tumors were weighed. Tumor tissues were embedded in paraffin and then sectioned for H\&E staining, a TUNEL assay, and IHC analysis to detect the expression of Bax, Bcl-2, Caspase-9, Caspase-3, and Cytc.

\section{Immunohistochemistry}

Tumors excised from mice were fixed in $10 \%$ formalin, embedded in paraffin, and cut into 4-mm sections. Deparaffinized tumor sections were treated with $3 \% \mathrm{H}_{2}$ $\mathrm{O}_{2}$ for $10 \mathrm{~min}$ to block endogenous peroxidases and incubated with $5 \%$ blocking serum (goat serum) at room temperature for $30 \mathrm{~min}$. After blocking, the slides were incubated with polyclonal rabbit anti-Caspase-3 (1:200 dilution; Proteintech, America), anti-Caspase-9 (1:100 dilution; Proteintech, Wuhan, China), anti-Bcl-2 (1:200 dilution; Proteintech, America), anti-Bax (1:200 dilution; Proteintech, Wuhan, China), and anti-Cytc (1:250 dilution; ABCAM, Britain) overnight at $4{ }^{\circ} \mathrm{C}$. The sections were then incubated for $1 \mathrm{~h}$ with a biotin labeled secondary antibody, followed by avidinperoxidase reagent and 3,3'-diaminobenzidine (DAB; Fuzhou, China) substrate. After hematoxylin counterstaining and dehydration, the sections were sealed with cover slips. Finally, the stained setions were observed under a microscope.

\section{Apoptosis Detection by TUNEL Assay}

Mice tissue sections were collected and paraffinized. They were then dewaxed and hydrated. Next, the sections were reacted for $15-20 \mathrm{~min}$ with ProteinaseK solution at $37^{\circ} \mathrm{C}$, and the positive sections was treated with $100 \mu \mathrm{L}$ DNaseI reaction solution. Each sample was emersed in $100 \mu \mathrm{L}$ TdT enzyme reaction solution, in darkness and wetness at $37^{\circ} \mathrm{C}$ for $1 \mathrm{~h}$. Subsequently, $100 \mu \mathrm{L}$ Streptavidin-HRP was added and incubated for $30 \mathrm{~min}$ at $37^{\circ} \mathrm{C}$. The sections were then colored with $\mathrm{DAB}$, and then were sealed with cover slips after hematoxylin counterstaining and dehydrated. The level of apoptosis was examined under a microscope and recorded.

\section{Statistical Analysis}

Statistical analyses were performed using SPSS software 16.0. All experiments were performed at least three times and the data are expressed as the mean \pm standard deviation. Comparisons between two groups were performed using Student's $t$-tests. Multiple groups were compared using one-way analyses of variance and Tukey's post-hoc test. $P<0.05$ was considered statistically significant difference. 


\section{Results}

\section{Cell Proliferation Effects of Different Doses of VitK3 and UVB and the Combination of VitK 3 and UVB}

Treatment with different concentrations $(0,30,45,60$, and 100 $\mu \mathrm{mol} / \mathrm{L}$ ) of VitK3 for $24 \mathrm{~h}$ reduced cell growth in a dosedependent manner. The median inhibitory concentration (IC50) of VitK3 was $40 \mu \mathrm{mol} / \mathrm{L}$ in A431 cells producing a corresponding inhibition rate of $50.7 \% \pm 2.88 \%$ (Supplementary Figure A). Similarly, cell irradiation with different doses of UVB $\left(0,0.5,1.0,1.5\right.$, and $\left.2.0 \mathrm{~J} / \mathrm{cm}^{2}\right)$ for $24 \mathrm{~h}$ also showed a reduction in tumor cell growth in a dosedependent manner. The half inhibitory dose of UVB was $0.8 \mathrm{~J} /$ $\mathrm{cm}^{2}$, yielding an inhibition rate of $49.85 \% \pm 3.02 \%$ in A431 cells. The inhibition rate was moderate at $1.5 \mathrm{~J} / \mathrm{cm}^{2}$ (Supplementary Figure B). These results indicated that VitK3 and UVB reduced tumor cell viability in a dosedependent manner.

To compare the individual effect of VitK 3 and UVB on the proliferation of A431 cells with the effects of VitK3 combined with UVB, the median inhibitory concentration of VitK3 $(40 \mu \mathrm{mol} / \mathrm{L})$ and the half inhibitory dose of UVB $\left(0.8 \mathrm{~J} / \mathrm{cm}^{2}\right)$ were chosen as the treatment concentration and light dose, respectively. Based on the CCK-8 assay, there was no significant difference in cell proliferation inhibition among the three treatment groups at $12 \mathrm{hrs}(\mathrm{P}>0.05)$. However, at $24 \mathrm{hrs}$, the inhibition rate in the VitK3-UVB group was significantly higher than that of the VitK 3 group ( $p$ $=0.002)$ and the UVB group $(p=0.0082)$. This phenomenon is the same for $24 \mathrm{hrs}$ and $48 \mathrm{hrs}(\mathrm{P}<0.05)$. These results demonstrate that the combination of VitK 3 and UVB has a superior inhibitory effect on proliferation of A431 cells than the VitK3 and the UVB alone (Figure 1B).

\section{Effect of VitK3, UVB, and VitK3-UVB on Apoptosis of A43I Cells}

Flow cytometry analysis showed that the apoptosis rate of A431 cells in control, VitK3, UVB, and VitK3-UVB groups was $8.36 \% \pm 0.005 \%, 28.00 \% \pm 1.07 \%, 25.50 \% \pm 0.50 \%$, and $47.28 \% \pm 1.40 \%$, respectively. The VitK3, UVB, or VitK3-UVB groups significantly induced apoptosis. However, the level of apoptosis was significantly higher in the VitK3-UVB treatment group than in VitK3 or UVB treatment groups $(\mathrm{P}<0.01)$. Besides, there was a statistically significant difference between the rate of apoptosis in the VitK3 and UVB treatment groups $(\mathrm{P}<0.05)$
(Figure 2). These results indicate that VitK3 combined with UVB can enhance the rate of apoptosis of A431 cells.

\section{Morphological Characteristics of Apoptosis}

To investigate the morphological features of apoptosis, we grouped A431 cells into a control group (A431), VitK3 drug group $(40 \mu \mathrm{mol} / \mathrm{L})$, UVB group $\left(800 \mathrm{~J} / \mathrm{cm}^{2}\right)$, and VitK3-UVB group $\left(40 \mu \mathrm{mol} / \mathrm{L}+800 \mu \mathrm{mJ} / \mathrm{cm}^{2}\right)$. Examination of the samples under a fluorescence microscope after Hoechst 33258 staining, revealed that the cells in the control group were evenly stained and there was no obvious apoptotic fluorescent signal. However, the A431 cells in the experimental groups, (VitK3, UVB, or VitK3UVB group), were significantly reduced compared with the control group. In particular, the reduction was more pronounced in the VitK3-UVB group (Figure 3). Some of the changes observed include cellular reduction, bright staining of the nucleus, and nuclear dispersion.

\section{Effect of VitK3-UVB on Cell Apoptosis via a Mitochondria-Mediated Apoptotic Pathway}

The production of ROS and the depolarization of the mitochondrial membrane potential (MMP) are good indicators of on-going cell death. ${ }^{16}$ Flow cytometry analysis was used to detect changes in the ROS level and MMP in A431 cells treated with a combination of VitK3-UVB. The results showed that ROS production rates in A431 cells in the control, VitK3, UVB, and VitK3-UVB groups were $2.17 \% \pm 0.17 \%, 15.82 \%$ $\pm 1.50 \%, 7.09 \% \pm 0.42 \%$, and $25.53 \% \pm 0.60 \%$, respectively (Figure 4A), whereas the incidence of MMP depolarization was $4.65 \% \pm 0.14 \%, 18.40 \% \pm 0.72 \%, 12.53 \% \pm 0.48 \%$, and $28.72 \% \pm 0.73 \%$, respectively (Figure $4 \mathrm{~B}$ ). The treatment groups showed increased production of ROS and depolarization of the MMP compared with the control group. Overall the VitK3-UVB group had the highest ROS production followed by UVB group and VitK3 group. An increase in ROS and MMP depolarization is an indication that the binding of VitK3 to UVB induces apoptosis by activating the mitochondrial pathway.

\section{Effect of VitK3-UVB on the Expression of Apoptotic Proteins and Anti-Apoptotic Proteins}

Western blot analysis revealed that the expression of apoptotic protein-Bax, Caspase-9, Caspase-3, and Cytc was higher in the VitK3-UVB treatment group than in the 


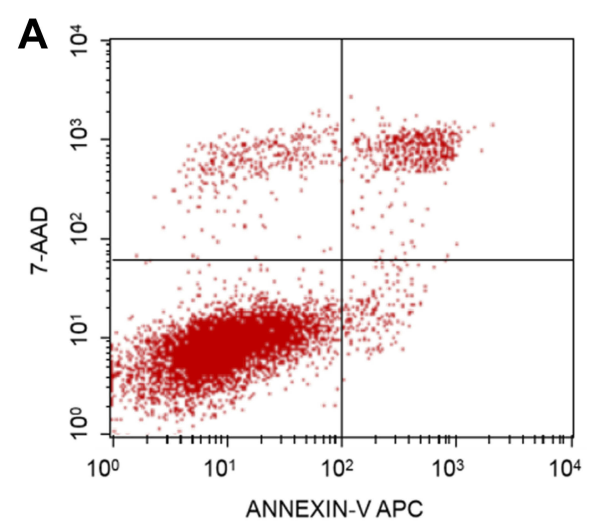

\section{Control}

\begin{tabular}{cc} 
Quad & \%Gated \\
\hline UL & 4.12 \\
UR & 6.24 \\
LL & 87.52 \\
LR & 2.12
\end{tabular}

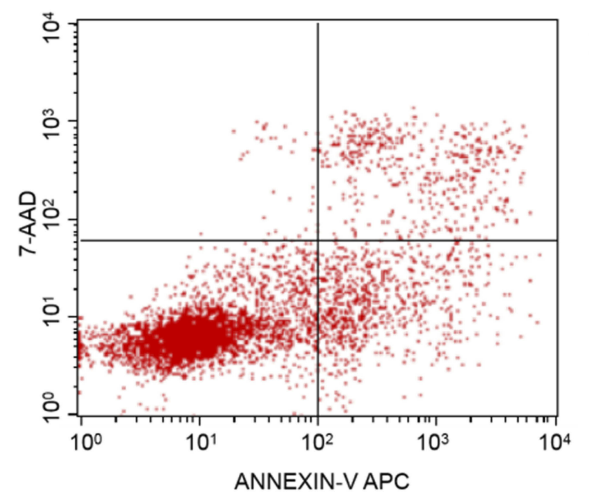

UVB

\begin{tabular}{cc} 
Quad & \%Gated \\
\hline UL & 0.81 \\
UR & 9.31 \\
LL & 74.07 \\
LR & 15.81
\end{tabular}

B

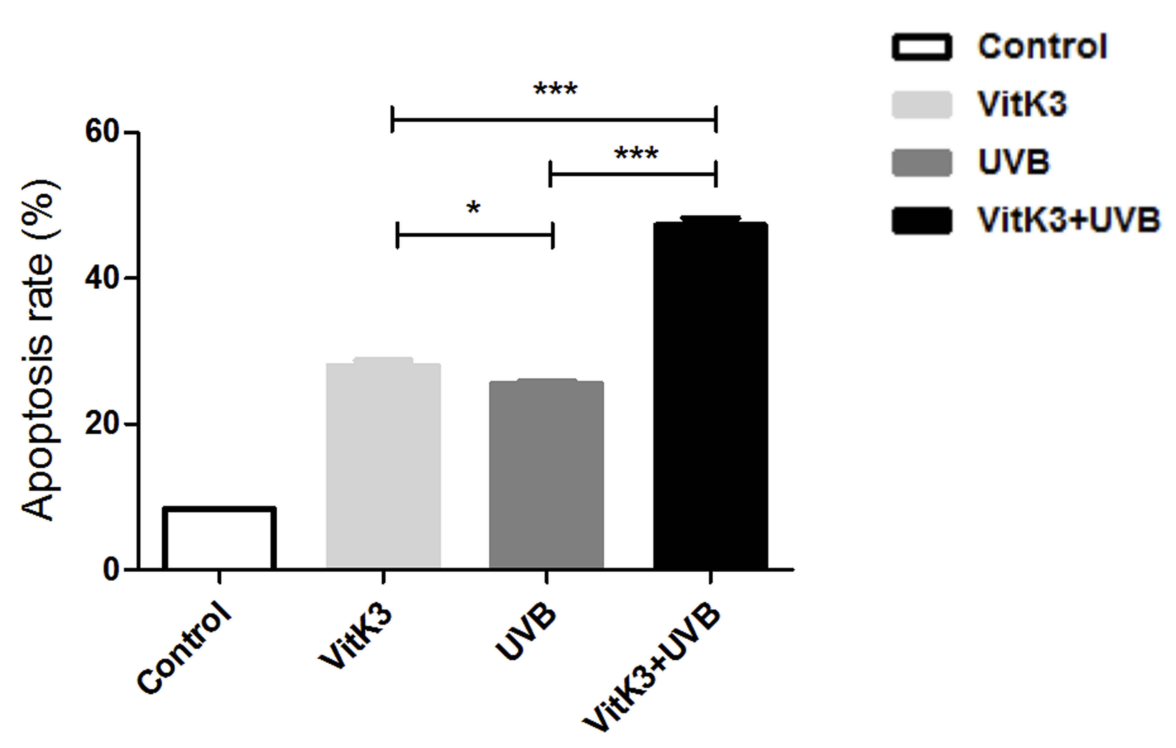

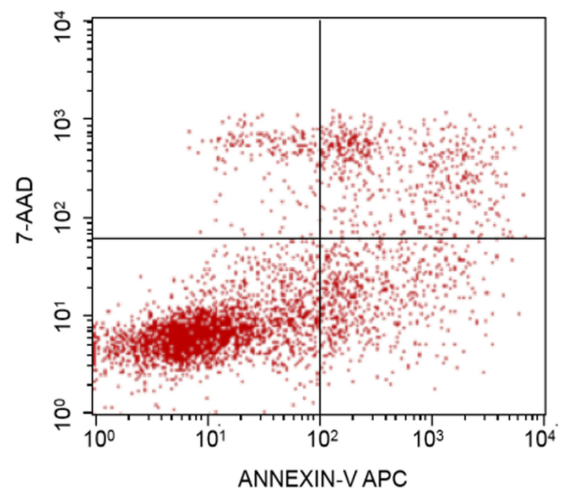

VitK 3

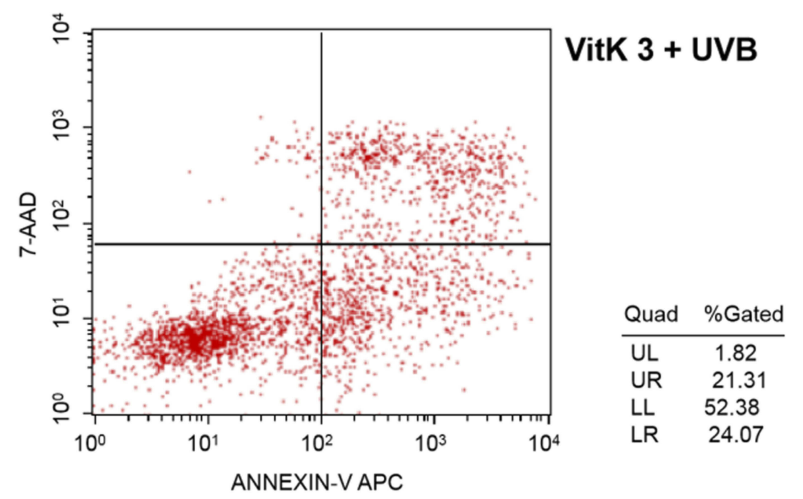

Figure 2 Flow cytometry analysis of the control, VitK3 $(40 \mu \mathrm{mol} / \mathrm{L})$, UVB group $\left(0.8 \mathrm{~J} / \mathrm{cm}^{2}\right)$, and VitK3-UVB group $\left(40 \mu \mathrm{mol} / \mathrm{L}+0.8 \mathrm{~J} / \mathrm{cm}^{2}\right)(\mathbf{A})$. The apoptosis rate of $A 43 \mathrm{I}$ cells in the four groups $(\mathbf{B})$. Data are shown as mean \pm standard deviations. $* \mathrm{P}<0.05, * * * \mathrm{P}<0.001$.

control, VitK3, and UVB groups. Further, there was a statistically significant difference in the expression of anti-apoptotic protein-Bcl-2 which was weaker in the
VitK3-UVB treatment group compared with the control, VitK3, and UVB groups. In addition, the expression of Caspase-9 was significantly higher in VitK3 group than in 

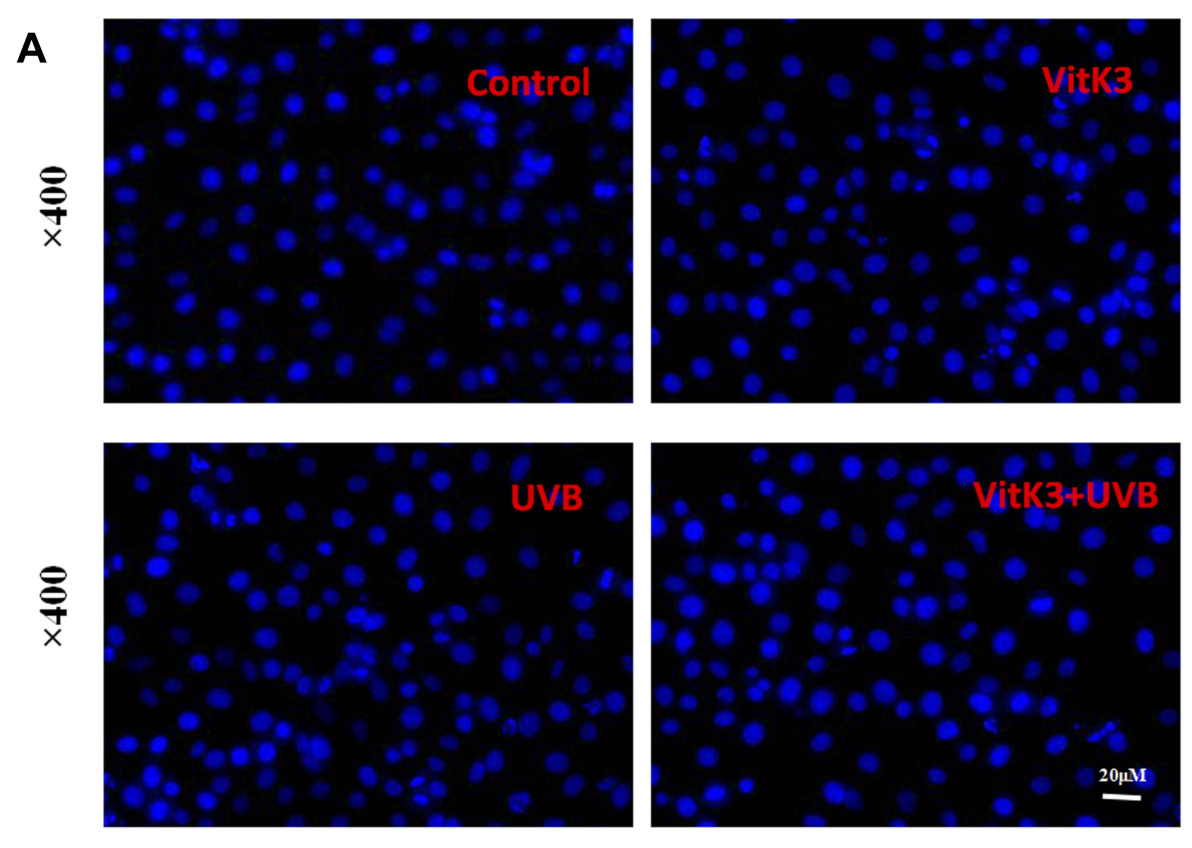

B

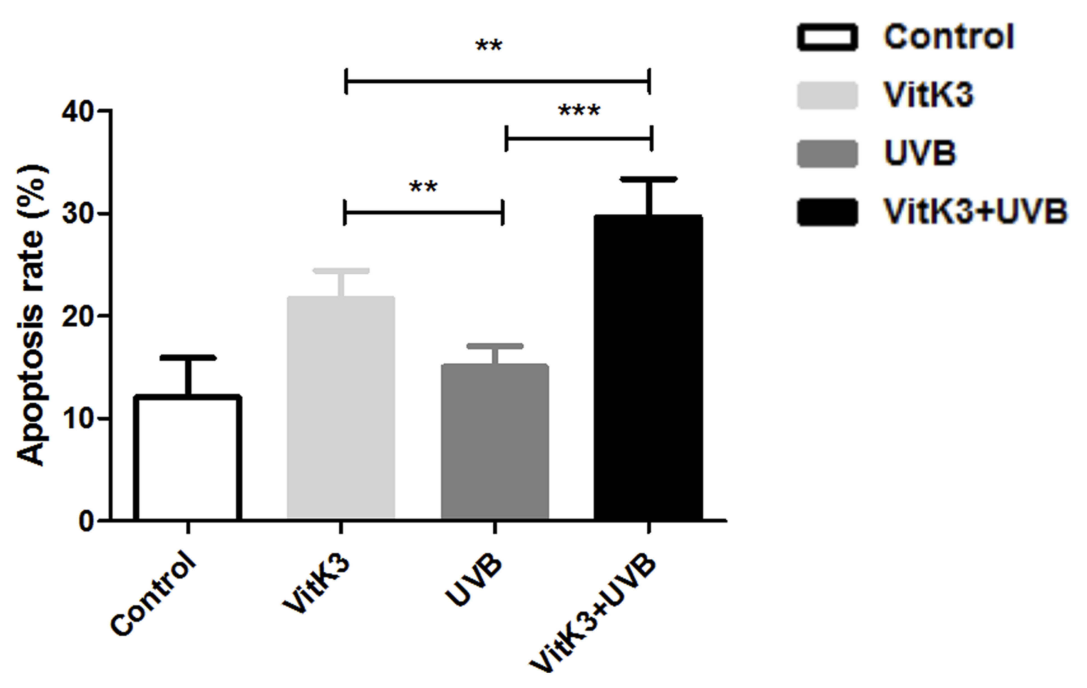

Figure 3 Morphological characteristics of apoptosis after treatment with vitamin K3 (40 $\mu \mathrm{mol} / \mathrm{L}), \mathrm{UVB}\left(800 \mathrm{~mJ} / \mathrm{cm}^{2}\right)$, or VitK3-UVB $\left(40 \mu \mathrm{mol} / \mathrm{L}+800 \mathrm{~mJ} / \mathrm{cm}^{2}\right)$ on A43I cells (A). The apoptosis rate of A43I cells in these four groups (B). Data are shown as mean \pm standard deviations. $* * \mathrm{P}<0.0 \mathrm{I}$, $* * * \mathrm{P}<0.00 \mathrm{I}$.

the UVB group, while the expression of Cytc was significantly higher in the UVB group than in VitK3. There was no difference in other apoptosis-related proteins in the VitK3 group and UVB group (Figure 5). These results indicate that the combination of VitK3 and UVB enhance the expression of apoptotic proteins and reduce the expression of the anti-apoptotic protein in A431 cells. The findings further demonstrate that the VitK3-UVB treatment group can induce increased cell apoptosis compared with the control, VitK3, and UVB groups.

\section{Effect of VitK3 Combined with UVB on Tumor Growth and Apoptosis in vivo}

The effect of VitK3 combined with UVB on the proliferation and apoptosis of A431 cells was investigated in tumor xenograft mouse models (Figure 6A) as well as the $\mathrm{H}$

$\&$ E staining (Figure $6 \mathrm{~B}$ ). The results indicated that the tumors in the VitK3-UVB group exhibited a significantly reduced tumor growth capacity compared with the control group $(\mathrm{P}<0.001)$, UVB group $(\mathrm{P}=0.01)$, and VitK3 group $(\mathrm{P}=0.001)$ (Figure $6 \mathrm{C}$, Table 1$)$.The tumor mass 

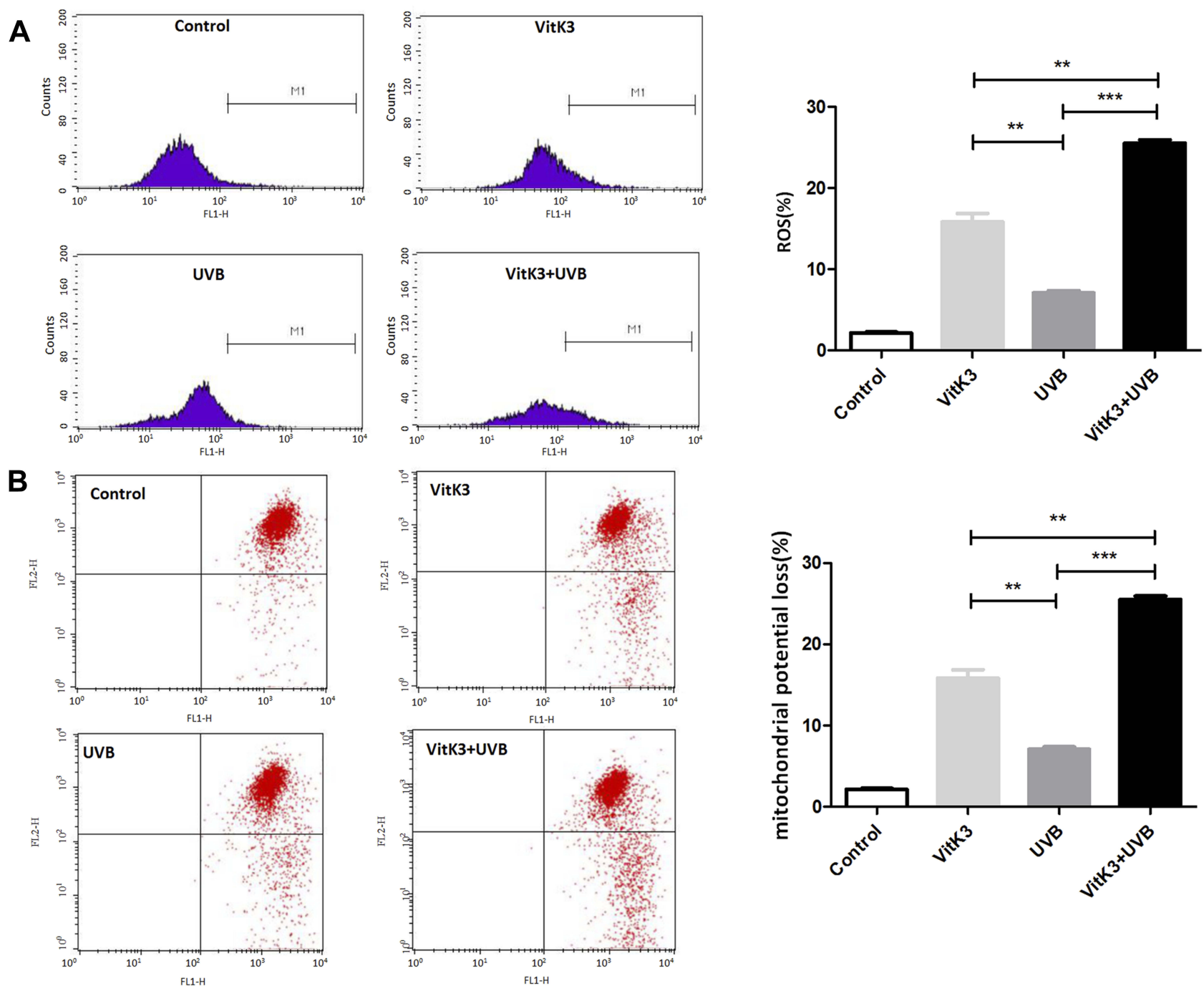

Figure 4 Treatment of A43I cells with VitK3 $(40 \mu \mathrm{mol} / \mathrm{L})$, UVB $\left(800 \mathrm{~mJ} / \mathrm{cm}^{2}\right)$, and VitK3 combined with UVB $\left(40 \mu \mathrm{mol} / \mathrm{L}+800 \mathrm{~mJ} / \mathrm{cm}^{2}\right)$, the intracellular ROS content $(\mathbf{A})$ and changes in mitochondrial membrane potential $(\mathbf{B})$ detected by flow cytometry. Data are shown as mean \pm standard deviations. $* * \mathrm{P}<0.0 \mathrm{I}, * * * \mathrm{P}<0.00 \mathrm{I}$.

in the VitK3 group was similar to that of the UVB group $(\mathrm{P}=0.065)$. The tumor volume in the VitK3-UVB group was lower than that in the VitK3, UVB, and VitK3 groups which showed no significant difference $(\mathrm{P}=0.956)$ (Figure 6D, Table 1). The tumor weight inhibitory rate in the VitK3, UVB, and VitK3-UVB groups reached $7.80 \%$, $15.07 \%$, and $26.42 \%$, respectively. In addition, the proliferation rate $(\mathrm{T} / \mathrm{C})$ in the VitK3, UVB, and the VitK3-UVB groups was $93.46 \%, 85.44 \%$, and $77.46 \%$, respectively (Figure 6E, Table 1). TUNEL assay revealed that the apoptosis rate of the VitK3-UVB, VitK3, and UVB groups was higher than that of the control group (Figure 6F). Immunohistochemical staining revealed that the level of Caspase-3, Caspase-9, Bax, and Cytc expression was significantly increased and level of Bcl-2 expression was decreased in the VitK3-UVB group when compared with the control, VitK3, and UVB groups (Figure 6G).

\section{Discussion}

cSCC is a common non-melanoma skin malignancy originating from the keratinocytes of skin epidermis or appendages and it is also known as Squamous cell carcinoma. cSCC typically occurs in the exposed parts of the body, such as the head, face, and neck. ${ }^{17}$ Moreover, it is the second-largest cutaneous malignant tumor after basal cell carcinoma; however, due to its high early metastatic rate, it is the leading cause of death among the nonmelanoma skin tumors. ${ }^{17-20}$

Surgery is the most widespread form of treatment used for $\mathrm{cSCC}$ with a limited range of lesions. However, since 


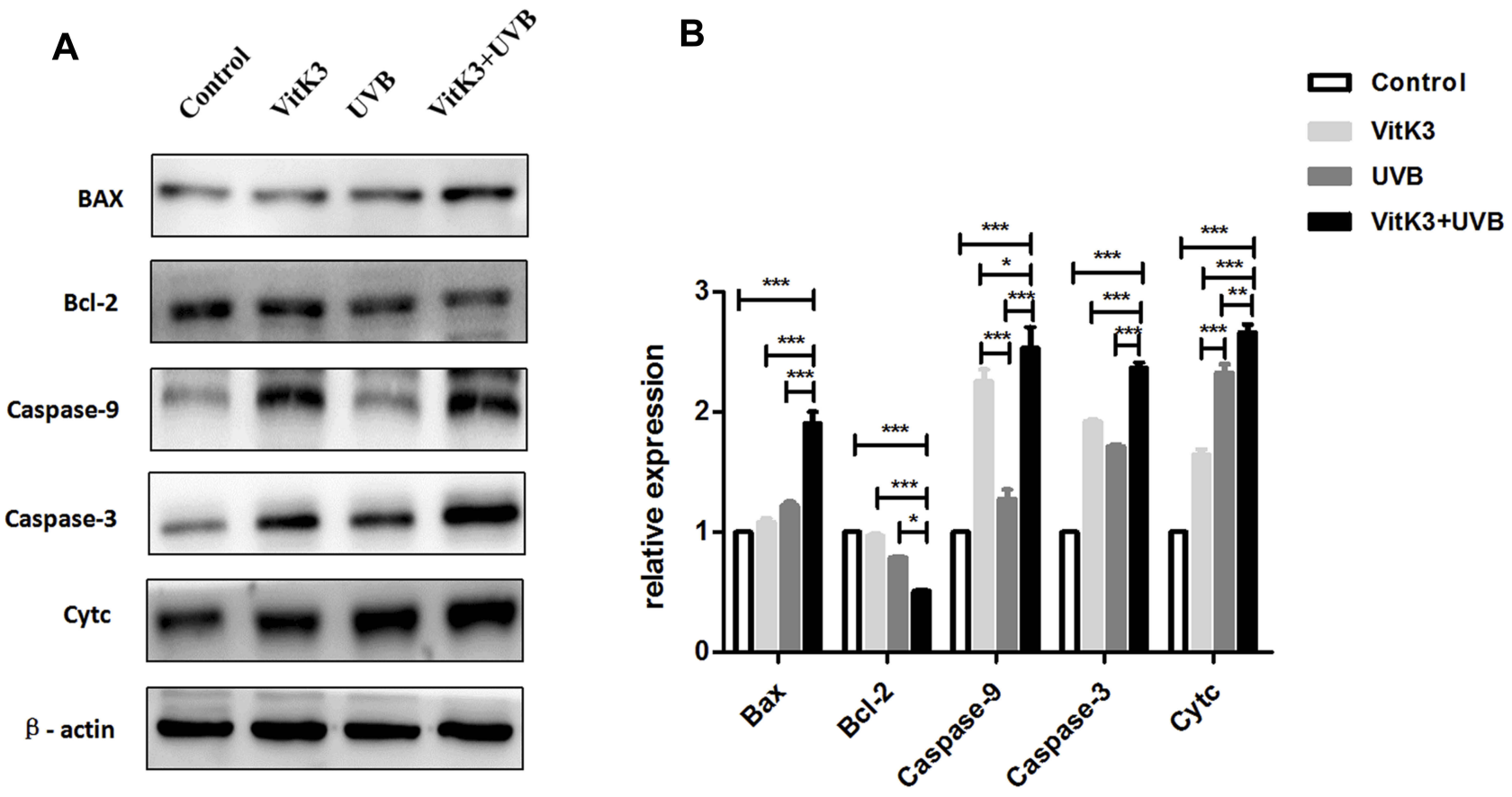

Figure 5 Western blot analysis of the expressed apoptotic proteins Caspase-3, Caspase-9, Bax, and Cytc and anti-apoptotic proteins, Bcl-2 (A). Quantitation of changes in band densities was performed by Imagej software, and the changes expressed as a ratio relative to levels in untreated cells set to I.0 (B). Data are shown as mean \pm standard deviations. $* \mathrm{P}<0.05$, $* * \mathrm{P}<0.01$, $* * * \mathrm{P}<0.001$

cSCC often occurs on the head and face, post-operative scars and facial deformities can seriously affect the patients' physical and mental health. In addition, due to the complexity of the facial structure, the unclear margins of the surgical area can cause tumor recurrence. Consequently, combined radiotherapy is often used for cSCC patients who cannot tolerate surgery or those who are prone to recurrence after cSCC resection of the head and face. In addition, shallow $\mathrm{X}$-ray and low megavolt electron beam methods are mainly used in radiotherapy for these patients. Drug therapy often consists of retinoic acid, cisplatin, interferon, and targeted drugs inhibiting the epidermal growth factor. Although gene therapy has been used to implant exogenous genes to correct for genetic defects, the high cost limits its popularity. ${ }^{5}$ Therefore, increased attention has focused on the search for an economical and effective treatment method for non-melanoma skin cancer. Compared with traditional therapies, PDT has several advantages (e.g., has a wide treatment application range, does not exhibit drug resistance, does not require repeated radiation, causes minimal injuries to the surrounding normal tissue, and causes minimal side effects). ${ }^{19}$

Photosensitive drugs enter the body where they accumulate in the lesions, they absorb energy upon irradiation using a laser of a specific wavelength, and this produces a series of photochemical and photobiological reactions. These excite the triplet oxygen molecule ${ }^{3} \mathrm{O}_{2}$ into active singlet oxygen ${ }^{1} \mathrm{O}_{2}$, and generation of other free radicals. The active singlet oxygen ${ }^{1} \mathrm{O}_{2}$ and other free radicals are strong oxidants, which bind to the stroma in the target tissue, causing it to be strongly oxidized, the cell loses its metabolic function, the cell membrane dissolves, enzymes are inactivated, proteins are denatured, and the tumor cells die due to these consequential irreversible damages. ${ }^{20}$ Photosensitizers are important in PDT and traditional photosensitizers are expensive and lack antitumor effects. However, second-generation photosensitizers such as 5-aminolaevulinic acid (ALA) have been reported to accumulate in the tumor tissue and upon activation by laser irradiation at a specific wavelength they produce singlet oxygen or other free radicals. These radicals can damage the blood vessels of tumor tissues, induce the activation of the inflammatory response, and use other mechanisms to induce tumor damage by apoptosis or necrosis. ${ }^{21}$ However, ALA also has some disadvantages (e.g., long-term retention in the body, limited lightshielding time, limited penetration depth, poor water solubility, hysteretic phototoxicity, and high cost), which limits its use in treatment. The lesions are also prone to recurrence and this restricts of its use in PDT. Therefore, the 
A

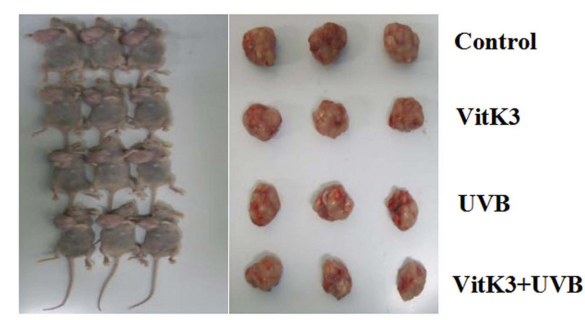

B
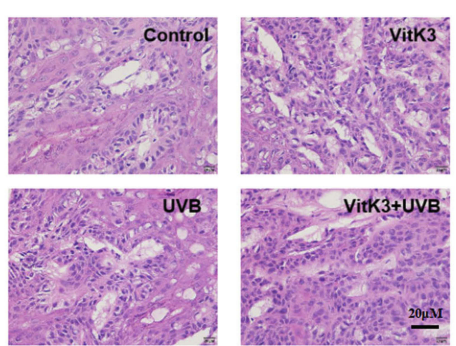

C

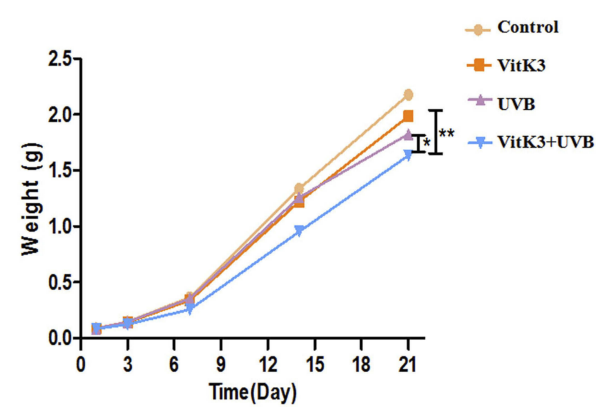

D

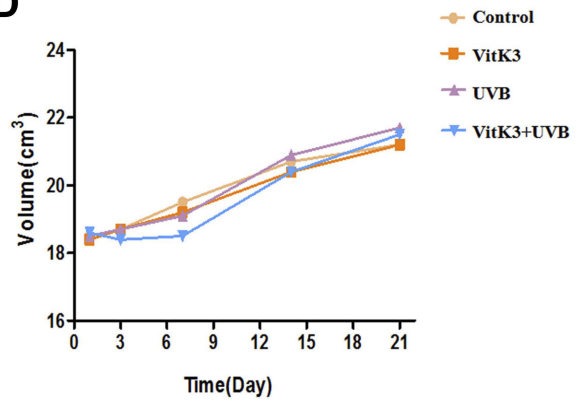

E
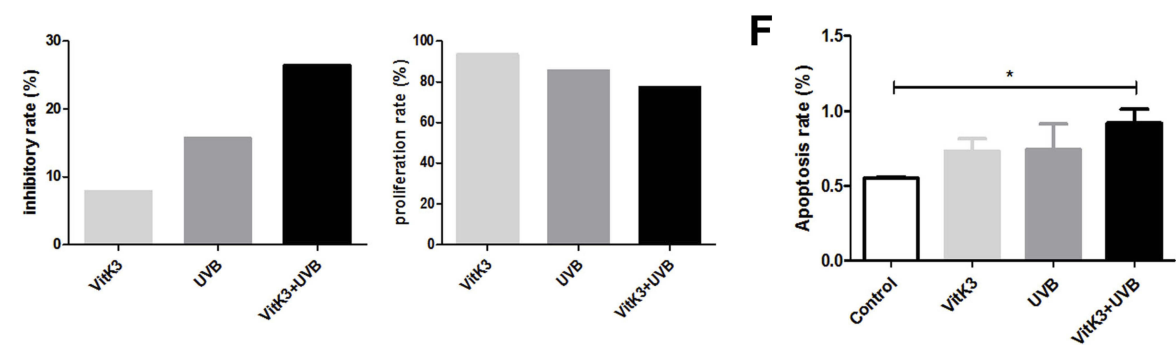

G
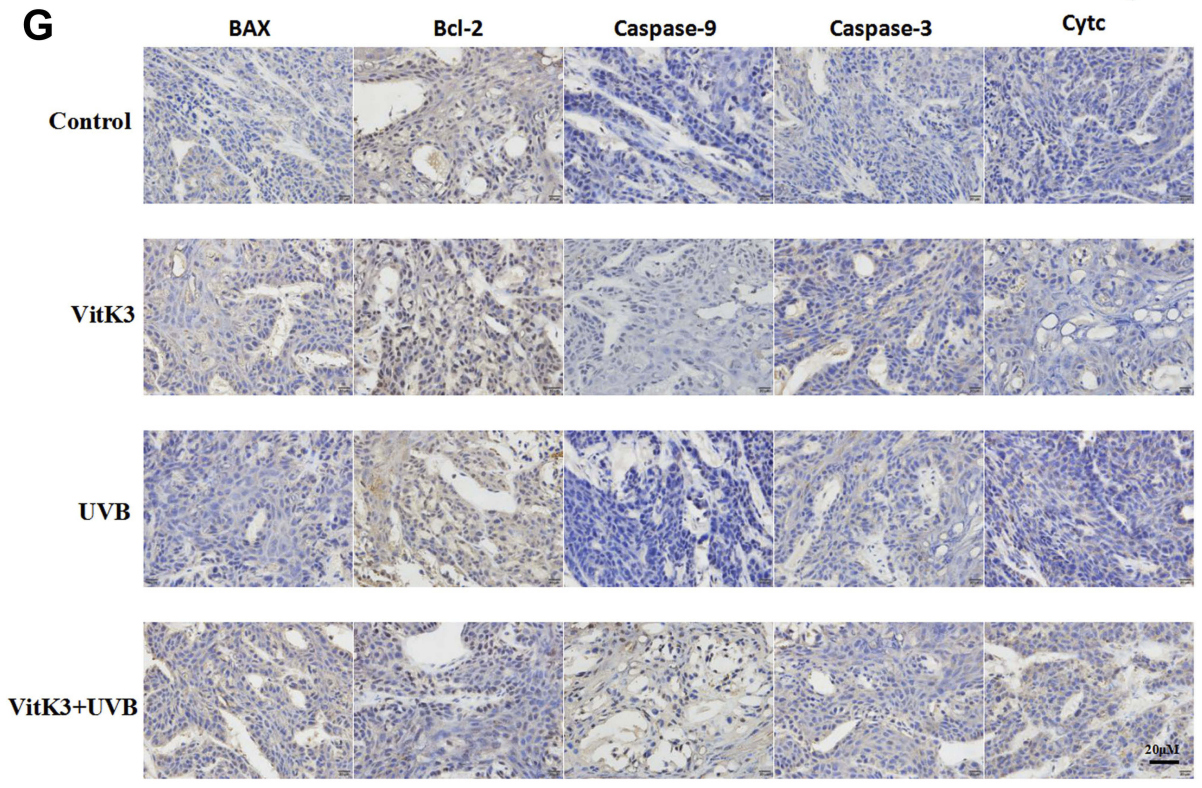

Figure 6 General observations of the subcutaneous tumors in nude mice ( $n=5$ in each group) (A) and H\&E staining in each group (B). The weight and volume of the xenograft tumors in the control model, VitK3, UVB, and VitK3-UVB groups of the human cutaneous squamous A43I cell line (C and D). The tumor weight and tumor proliferation rate (T/C) in the VitK3, UVB, and VitK3-UVB groups (E). The apoptosis rate of each group detected using a TUNEL assay (F). The level of Bax, Bcl2, Caspase-9, Caspase-3, and Cytc protein expression assessed using IHC in the xenograft tumor sections in the control, VitK3, UVB, and VitK3-UVB groups (G). Data are shown as mean \pm standard deviations. *P $<0.05$, **P $<0.01$.

development of new photosensitizers with high efficiency decreased toxicity, and low cost is important in promoting PDT in cancer treatment.
VitK3 has been reported to be a radiosensitizer for hypoxic tumors and can also be used in combination with chemotherapeutic drugs. ${ }^{14}$ The antitumor effect of VitK3 can 
Table I Inhibitory Effect of VitK3, UVB, and VitK3-UVB on the Growth of the Human Cutaneous Squamous A43I Cell Line in Nude Mice

\begin{tabular}{|l|l|l|l|l|l|l|}
\hline Group & Tumor Volume $\left.\mathbf{( c m}^{\mathbf{3}}\right)$ & P & T/C & Tumor Weight $\mathbf{( g )}$ & P & Inhibition Ratio \\
\hline NC & $2.179 \pm 0.109$ & 0.956 & - & $1.94 \pm 0.09$ & $<0.001$ & - \\
Vitk3 & $1.984 \pm 0.106$ & & $93.46 \%$ & $1.79 \pm 0.08$ & $7.80 \%$ \\
UVB & $1.826 \pm 0.076$ & & $85.44 \%$ & $1.65 \pm 0.09$ & $15.07 \%$ \\
Vitk3+UVB & $1.635 \pm 0.057$ & & $77.46 \%$ & $1.43 \pm 0.06$ & $26.42 \%$ \\
\hline
\end{tabular}

be achieved by the following three mechanisms: 1) generation of active oxygen through a redox cycle and a type II photoreaction; 2) conjugation of VitK3 with the thiol groups of proteins leading to the depletion of glutathione and changes in the intracellular $\mathrm{Ca}^{2+}$ concentrations; and 3) VitK3 arylation directly activates transcription factors and other proteins. ${ }^{22-24}$ Based on previous research studies, we hypothesized that VitK3 is an endogenous photosensitizer and a drug that can induce apoptosis of cancer cells by producing reactive oxygen, ${ }^{25}$ activating NF- $\mathrm{KB}, \mathrm{P} 53$ protein, c-JUN transcription factors, and causing mitochondrial depolarization which ultimately induces apoptosis. ${ }^{12}$ If VitK3 is combined with PDT, it is expected that this further promotes the production of ROS, activate the death receptor pathway, and promote the apoptosis of cancer cells.

In the present study, we found that different concentrations of VitK3 and UVB inhibited the proliferation of A431 cells at $24 \mathrm{~h}$. CCK-8 assay showed that VitK3 and UVB inhibited the growth of the tumor cells in a dosedependent manner, and VitK3-UVB was a better inhibitor of proliferation than the VitK3 and UVB alone.

Flow cytometry analysis confirmed that the apoptotic rate of the VitK3-UVB group was significantly higher than that of the control group, VitK3 group and UVB group. Hoechst33258 staining and observation of the A431 cells under a fluorescence microscope revealed typical changes in apoptosis which were more pronounced in the VitK3UVB group. This indicates that the VitK3, UVB, or VitK3UVB treatment can inhibit cell proliferation and induce apoptosis, while the anti-tumor effect of the VitK3-UVB treatment group is significantly enhanced.

The cellular apoptosis pathways are complex and diverse. PDT produces ROS which primarily initiates mitochondrial depolarization, causing changes in the mitochondrial transmembrane potential which induces apoptosis. ${ }^{26}$ In addition, changes in the ROS and MMP are closely related to apoptosis. Cells treated with PDT produce ROS, including singlet oxygen, oxygen-free radicals, and hydroxyl free radicals. When the production of ROS exceeds cellular clearance, mitochondrial function is affected. Some of the oxygen free radicals initiate mitochondrial depolarization, increase mitochondrial membrane permeability, as well as cause mitochondrial outer membrane swelling and rupture. This causes the cytochrome $\mathrm{C}$ to enter the cytoplasm from the mitochondrial membrane gap and activate the downstream caspase cascade which ultimately causes apoptosis. ${ }^{11,27,28}$ Kim et al report that UVB stimulates the generation of reactive oxygen species (ROS). ${ }^{29}$ This study found that the levels of ROS and MMP depolarization in the VitK3-UVB group was significantly higher than individual treatment groups. That is to say, VitK3-UVB induces apoptosis in A431 cells by increasing ROS and altering MMP.

Apoptosis is an active process, which involves the activation, expression, and regulation of a series of proteins as revealed by Western blot analysis. Among these processes, the cysteinyl aspartate specific proteinase (Caspase protein) and B-cell lymphoma 2 (Bcl-2) protein families play an important role in apoptosis signal transduction. Moreover, mitochondrial cytochrome $\mathrm{C}$ is closely related to the Caspase and Bcl-2 protein family. ${ }^{30}$ The Bcl-2 family member, Bax, allows some ions and small molecules (e.g., cytochrome c) to pass through the mitochondrial membrane, enter the cytoplasm, and induce apoptosis. However, Bcl-2 has an opposite effect where it inhibits the pore-forming activity of the Bax gene so that some small molecules cannot pass through freely, thereby protecting the cell from apoptosis. ${ }^{31}$ Due to the upstream regulatory mechanism of Caspase, the overexpression of $\mathrm{Bcl}-2$ inhibits Caspase activation. ${ }^{32}$ Cytochrome $\mathrm{C}$, which is released into the cytoplasm by the mitochondria, is assembled into an apoptotic complex (apoptosome) by binding to Apaf-1. In addition, Caspase-9 is recruited and activated by the apoptosome, which activates Caspase-3, and eventually lead to apoptosis. ${ }^{33-35}$ In this study, the expression of apoptotic proteins and antiapoptotic proteins in each group revealed that the combination of VitK3 and UVB can induce increased cell apoptosis compared with the VitK3 and UVB alone.

In vivo experiments revealed that mice treated with VitK3-UVB inhibited tumor growth and increased the 
tumor inhibitory rate. Besides, the expression levels of apoptotic proteins increased whereas the anti-apoptotic protein decreased in the VitK3-UVB group compared with the control, VitK3, and UVB groups. This demonstrates that UVB combined with VitK3 can induce apoptosis in cSCC.

This study demonstrates the ability of VitK 3 to function as a potential photosensitizer when combined with UVB to cause tumor damage at the cellular level. Further, this study reveals that a combination of VitK3 with UVB inhibits tumor cell proliferation and also induces apoptosis of A431 cells both in vitro and in vivo. These findings suggest a new treatment approach to non-melanoma skin cancers.

\section{Acknowledgments}

This work was supported by a grant from "Project of Cultivating the Innovation of Postgraduate Students" (SJCX18-0705), Jiangsu Province, China and the China the Science and Technology Project of Xuzhou city (No. Kh17044)

\section{Disclosure}

The authors declare that they have no conflicts of interest.

\section{References}

1. Nehal KS, Bichakjian CK. Update on keratinocyte carcinomas. N Engl J Med. 2018;379(4):363-374.

2. Asgari MM, Efird JT, Warton EM, Friedman GD. Potential risk factors for cutaneous squamous cell carcinoma includeoral contracetives: results of a nested case-control study. Int J Environ Res Public Health. 2010;7(2):427-442. doi:10.3390/ijerph7020427

3. Rogers HW, Weinstock MA, Feldman SR, Coldiron BM. Incidence estimate of nonmelanoma skin cancer (keratinocyte carcinomas) in the US population, 2012. JAMA Dermatol. 2015;151(10):1081-1086. doi:10.1001/jamadermatol.2015.1187

4. Janus JM, O'Shaughnessy RFL, Harwood CA, Maffucci T. Phosphoinositide 3-kinase-dependent signalling pathways in cutaneous squamous cell carcinomas. Cancers (Basel). 2017;9(7):E86.

5. Bander TS, Nehal KS, Lee EH. Cutaneous squamous cell carcinoma: updates in staging and management. Dermatol Clin. 2019;37 (3):241-251. doi:10.1016/j.det.2019.03.009

6. Gong H, Chao Y, Xiang J, et al. Hyaluronidase to enhance nanoparticle-based photodynamic tumor therapy. Nano Lett. 2016;16(4):2512-2521. doi:10.1021/acs.nanolett.6b00068

7. Kumar M, Curtis A, Hoskins C. Application of nanoparticle technologies in the combat against anti-microbial resistance. Pharmaceutics. 2018;10(1):E11.

8. Dolmans DE, Fukumura D, Jain RK. Photodynamic therapy for cancer. Nat Rev Cancer. 2003;3(5):380-387. doi:10.1038/nrc1071

9. McEwan C, Nesbitt H, Nicholas D. Comparing the efficacy of photodynamic and sonodynamic therapy in non-melanoma and melanoma skin cancer. Bioorg. Med. Chem. 2016;24(13):3023-3028. doi:10.10 16/j.bmc.2016.05.015

10. Gomes AT, Neves MG, Cavaleiro JAS. Cancer, photodynamic therapy and porphyrin-type derivatives. An Acad Bras Cienc. 2018;90(1 Suppl 2):993-1026. doi:10.1590/0001-3765201820170811
11. Abdi A, Sadraie H, Dargahi L, Khalaj L, Ahmadiani A. Apoptosis inhibition can be threatening in A $\beta$-induced neuroinflammation, through promoting cell proliferation. Neurochem Res. 2011;36 (1):39-48. doi:10.1007/s11064-010-0259-3

12. Bonilla-Porras AR, Jimenez-Del-Rio M, Velez-Pardo C. Vitamin K3 and vitamin $\mathrm{C}$ alone or in combination induced apoptosis in leukemia cells by a similar oxidative stress signaling mechanism. Cancer Cell Int. 2011;11:19. doi:10.1186/1475-2867-11-19

13. Kitano $\mathrm{T}$, Yoda $\mathrm{H}$, Tabata $\mathrm{K}$, et al. Vitamin $\mathrm{K} 3$ analogs induce selective tumor cytotoxicity in neuro blastoma. Biol Pharm Bull. 2012;35(4):617-623. doi:10.1248/bpb.35.617

14. Bergeron F, Klarskov K, Hunting DJ, Wagner JR. Near-UV photolysis of 2-methyl-1,4-naphthoquinon-DNA duplexes: characterization of reversible and stable interstrand cross-links between quinone and adenine moieties. Chem Res Toxicol. 2001;20(5):745-756. doi:10.10 21/tx6002968

15. Xu F, Jaroslav G. Inactivation of bacteria via photosensitization of vitamin K3 by UV-alight. FEMS Microbiol Lett. 2014;358 (1):98-105. doi:10.1111/1574-6968.12544

16. Petit PX, Lecoeur H, Zorn E, Dauguet C, Mignotte B, Gougeon ML. Alterations in mitochondrial structure and function are early events of dexamethasone-induced thymocyte apoptosis. J Cell Biol. 1995;130 (1):157-167. doi:10.1083/jcb.130.1.157

17. Feller L, Khammissa RAG, Kramer B, Altini M, Lemmer J. Basal cell carcinoma, squamous cell carcinoma and melanoma of the head and face. Head Face Med. 2016;12:11. doi:10.1186/s13005-016-0106-0

18. Tufaro AP, Chuang JC, Prasad N, Chuang A, Chuang TC, Fischer AC. Molecular markers in cutaneous squamous cell carcinoma. Int J Surg Oncol. 2011;2011:231475.

19. Shive ML, Coakley BJ, Bierman DF, et al. Use of 5-aminolevulinic acid and daylight photodynamic therapy for the treatment of actinic keratoses. Dermatol Surg. 2019;45(4):529-535. doi:10.1097/DSS.00 00000000001771

20. Bourré L, Rousset N, Thibaut S, Eléouet S, Lajat Y, Patrice T. PDT effects of m-THPC and ALA, phototoxicity and apoptosis. Apoptosis. 2002;7(3):221-230. doi:10.1023/A:1015391415150

21. Battah S, Hider RC, MacRobert AJ, Dobbin PS, Zhou T. Hydroxypyridinone and 5-aminolaevulinic acid conjugates for photodynamic therapy. J Med Chem. 2017;60(8):3498-3510. doi:10.1021/ acs.jmedchem.7b00346

22. Turner NA, Xia F, Azhar G, Zhang X, Liu L, Wei JY. Oxidative stress induces DNA fragmentation and caspase activation via the c-Jun $\mathrm{NH} 2$-terminal kinase pathway in $\mathrm{H} 9 \mathrm{c} 2$ cardiac muscle cells. $J \mathrm{Mol}$ Cell Cardiol. 1998;30(9):1789-1801. doi:10.1006/jmcc.1998.0743

23. Jamison JM, Gilloteaux J, Venugopal M, et al. Flow cytometric and ultrastructural aspects of the synergistic antitumor activity of vitamin C-vitamin K3 combinations against human prostatic carcinoma cells. Tissue Cell. 1996;28(6):687-701. doi:10.1016/S0040-8166(96)800 $72-3$

24. Venugopal M, Jamison JM, Gilloteaux J, et al. Synergistic antitumor activity of vitamin $\mathrm{C}$ and $\mathrm{K} 3$ on human urologic tumor cell lines. Life Sci. 1996;59(17):1389-1400. doi:10.1016/0024-3205(96)00466-3

25. Wondrak GT, Jacobson MK, Jacobson EL. Endogenous UVA-photosensitizers: mediators of skin photodamage and novel targets for skin photoprotection. Photochem Photobiol Sci. 2006;5 (2):215-237. doi:10.1039/B504573H

26. Topaloglu N, Guney M, Aysan N, Gulsoy M, Yuksel S. The role of reactive oxygen species in the antibacterial photodynamic treatment: photoinactivation vs proliferation. Lett Appl Microbiol. 2016;62 (3):230-236. doi:10.1111/lam.2016.62.issue-3

27. Lam M, Oleinick N, Nieminen A. Photodynamic therapy induced apoptosis in epidermoid carcinoma cells. Reactive oxygen species and mitochondrial inner membrane permeabilization. $J$ Biol Chem. 2001;276(50):47379-47386. doi:10.1074/jbc.M107678200

28. Loekshin RA. Programmed cell death: historyand future of a concept. J Soc Biol. 2005;1993(3):169-173. 
29. Kim JM, Kim SY, Noh EM, et al. Reversine inhibits MMP-1 and MMP-3 expressions by suppressing of ROS/MAPK/AP-1 activation in UV-stimulated human keratinocytes and dermal fibroblasts. Exp Dermatol. 2018;27(3):298-301. doi:10.1111/exd.13494

30. Martinou JC, Youle RJ. Mitochondria in apoptosis: bcl-2 family members and mitochondrial dynamics. Dev Cell. 2011;21 (1):92-101. doi:10.1016/j.devcel.2011.06.017

31. Kumar R, Vadlamudi RK, Adam L. Apoptosis in mammary gland and cancer. Endocr Relat Cancer. 2000;7(4):257-269. doi:10.1677/ erc. 0.0070257

32. Cheng EH, Kirsch DG, Clem RJ. Conversion of Bcl-2 to a Bax death effector by caspases. Science. 1997;278(5345):1966-1968. doi:10.11 26/science.278.5345.1966
33. Thornberry NA, Lazebnik Y. Caspases: enemies within. Science. 1998;281(5381):1312-1316. doi:10.1126/science.281.5381.1312

34. Saleh A, Srinivasula SM, Acharya S, Fishel R, Alnemri ES. Cytochrome $\mathrm{c}$ and dATP-mediated oligomerization of Apaf-1 is a prerequisite for procaspase-9 activation. J Biol Chem. 1999;274 (25):17941-17945. doi:10.1074/jbc.274.25.17941

35. Ruiz-Vela A, Korsmeyer SJ. Proapoptotic histone H1.2 induces CASP-3 and -7 activation by forming a protein complex with CYT c, APAF-1 and CASP-9. FEBS Lett. 2007;581(18):3422-3428. doi:10.1016/j.febslet.2007.06.049

\section{Publish your work in this journal}

OncoTargets and Therapy is an international, peer-reviewed, open access journal focusing on the pathological basis of all cancers, potential targets for therapy and treatment protocols employed to improve the management of cancer patients. The journal also focuses on the impact of management programs and new therapeutic

Submit your manuscript here: https://www.dovepress.com/oncotargets-and-therapy-journal agents and protocols on patient perspectives such as quality of life, adherence and satisfaction. The manuscript management system is completely online and includes a very quick and fair peer-review system, which is all easy to use. Visit http://www.dovepress.com/ testimonials.php to read real quotes from published authors. 